\title{
Radio Pulsar Sub-Populations (I) : The Curious Case of Nulling Pulsars
}

\author{
Sushan Konar ${ }^{1, *}$ and Uddeepta Deka ${ }^{1}$ \\ ${ }^{I}$ NCRA-TIFR, Pune, 411007, India. \\ ${ }^{2}$ Department of Physics \& Astrophysics, University of Delhi, 110007, India. \\ *Corresponding author. E-mail: sushan@ncra.tifr.res.in
}

\begin{abstract}
About 200 radio pulsars have been observed to exhibit nulling episodes - short and long. We find that the nulling fraction of a pulsar does not have any obvious correlation with any of the intrinsic pulsar parameters. It also appears that the phenomenon of nulling may be preferentially experienced by pulsars with emission coming predominantly from the polar cap region, and also having extremely curved magnetic fields.
\end{abstract}

Key words. radio pulsar-nulling-death-line.

\section{Introduction}

Fifty years of observations have yielded $~ 3000$ neutron stars, with diverse characteristic properties, which fall into three major categories, namely - a) the rotation powered, b) the accretion powered, and c) the internal-energy powered neutron stars; according to their mechanisms of energy generation (Kaspi 2010; Konar 2013; Konar et al. 2016; Konar 2017). Radio pulsars, which belong to the category of rotation powered pulsars (RPP), are strongly magnetized rotating neutron stars (mostly isolated or in non-interacting binaries) characterized by their short spin periods $(P \sim$ $\left.10^{-3}-10^{2} \mathrm{~s}\right)$ and large inferred surface magnetic fields $\left(B \sim 10^{8}-10^{15} \mathrm{G}\right)$. Powered by the loss of rotational energy, they emit highly coherent radiation (typically spanning almost the entire electromagnetic spectrum) which are observed as narrow emission pulses. The abrupt cessation of this pulsed emission for several pulse periods, observed in a small subset of radio pulsars, is known as the phenomenon of nulling - noticed for the first time by Backer (1970). Since then, close to two hundred radio pulsars have been observed to experience nulling. In this context, it needs to be noted that most of the $\sim 2600$ radio pulsars are neither monitored regularly, nor are searched for the presence of nulling. This would imply that two hundred is just a lower limit to the actual number of nulling pulsars. On the other hand, presence of nulling may also depend on the sensitivity of a given telescope (e.g. a telescope with a low sensitivity may consider a pulsar to be nulling when a similar (or same) pulsar might be detected in weak emission by an instrument with higher sensitivity), giving rise to an over-estimate of the number of nulling pulsars.

(c) Indian Academy of Sciences
In general, two parameters are used to quantify the phenomenon of nulling -

1. the nulling fraction (NF) - the total percentage fraction of pulses without detectable emission; and,

2. the null length (NL) - the duration of a given nulling episode.

Both NF and NL are observed to span a wide range while NF ranges from just a few to more than $90 \%$, NL can go from the simple case of single pulse nulls to the extreme situation of complete disappearance of pulsed emission for as long as a few years. Even though most pulsars are known to be characterised by a single value of NF (see the tables in Appendix A. for some contrary cases), neither NF nor NL can uniquely describe the behaviour of a nulling pulsar. It is well known that NL not only varies from one pulsar to another, but also from episode to episode for a given pulsar (Young et al. 2012). Moreover with increasing data it is becoming evident that two different pulsars having very different values of NL and totally different nulling behaviour can have the same average value of NF ( $\mathrm{Ga}-$ jjar, Joshi, \& Kramer 2012). For example, the long quiescent states of intermittent pulsars are in stark contrast to the longest known quiescence times of ordinary nulling pulsars, i.e., they differ in their nulling timescale by about five orders of magnitude - even when the $\mathrm{NF}$ values are similar for both cases.

A detailed discussion on different types of nulling behaviour can be found in Gajjar (2017) and references therein. Despite the wide variation in NL, the population does render itself to a broad classification, depending on the nature of nulling, as follows - 
1. Classical Nuller $(\mathrm{CN})$ - pulsars with mostly single (or just a few) pulse nulls, for example - J0837-4135, J2022+5154 (Gajjar, Joshi, \& Kramer 2012);

2. Intermittent Nuller (IN) - NL is longer, could be up-to a few hours combined with a longer period of activity, for example - J1717-4054 (Johnston et al. 1992), J1634-5107 (O'Brien et al. 2006), J1709-1640 (Naidu et al. 2018);

3. Intermittent Pulsar (IP) - NL can vary from days to years, for example - J1933+2421 (Kramer et al. 2006a), J1832+0029 (Lorimer et al. 2012), J1910+0517 \& J1929+1357 (Lyne et al. 2017);

4. Rotating Radio Transient (RRAT) - Discovered in 2006, the RRATs are characterised by their sporadic single pulse emissions (McLaughlin et al. 2006). Whether these can be considered to be part of the nulling fraternity is a contentious issue, which we plan to take up in a later study (Konar 2019).

The phenomenon of nulling is usually observed to be associated with other emission features, like the drifting of sub-pulses and mode changing (Wang, Manchester, $\&$ Johnston 2007). Certain other behavioural changes have also been seen in nulling pulsars. In J1933+2421 the spin-down rate has been observed to decrease in the inactive phase compared to the active phase, suggestive of a depletion in the magnetospheric particle outflow in the quiescent phase of the pulsar (Kramer et al. 2006b; Lyne et al. 2009). An exponential decrease in the pulse energy during a burst has also been seen in certain nulling pulsars (Rankin \& Wright 2008; Bhattacharyya, Gupta, \& Gil 2010; Li et al. 2012; Gajjar et al. 2014). Interestingly, nulling behaviour has not yet been observed in a millisecond pulsar (Rajwade et al. 2014), even though the cumulative study of this class of pulsars is close to $10^{3}$ years.

In general, two different classes of models are invoked to explain the phenomenon of nulling, explaining it to arise from - a) intrinsic causes or b) geometrical effects. Some of the models attributing nulling to an intrinsic cause are as follows -

- the loss of coherence conditions (Filippenko \& Radhakrishnan 1982);

- a complete cessation of primary particle production (Kramer et al. 2006b; Gajjar et al. 2014);

- changes in the current flow conditions in the magnetosphere (Timokhin 2010);
- a transition to a much weaker emission mode (or an extreme case of mode changing) (Esamdin et al. 2005; Wang, Manchester, \& Johnston 2007; Timokhin 2010; Young et al. 2014);

- time-dependent variations in an emission 'carousel model' (Deshpande \& Rankin 2001; Rankin \& Wright 2007); etc.

On the other hand, a variety of geometrical effects have also been suggested to explain nulling, like -

- the line-of-sight passing between emitting subbeams giving rise to 'pseudo-nulls' (Herfindal \& Rankin 2007; Herfindal \& Rankin 2009; Rankin \& Wright 2008);

- occurrence of various unfavourable changes in the emission geometry (Dyks, Zhang, \& Gil 2005; Zhang, Gil, \& Dyks 2007).

Detailed investigations of the nulling behaviour of individual pulsars and theoretical modeling of this phenomenon have been undertaken by many groups (Ritchings 1976, Rankin 1986; Biggs 1992; Wang, Manchester, \& Johnston 2007; Gajjar, Joshi, \& Kramer 2012). In many instances, nulling has been observed across a wide frequency range making it a broadband phenomenon (even though the exact value of NF reported appears to have large variation over observing frequencies). This is strongly suggestive of intrinsic changes being responsible for nulling rather than geometrical effects. Not surprisingly, many subscribe to the thought that nulling is of magnetospheric origin (Kramer et al. 2006b; Wang, Manchester, \& Johnston 2007; Lyne et al. 2010).

Therefore, it is important to look at the overall characteristics of the population of nulling pulsars in an effort to understand the origin of the phenomenon. A comprehensive list of nulling pulsars has recently been generated by Gajjar (2017) comprising of 109 objects. For the present work, we have done an extensive literature survey to extend and update that list. The number of nulling pulsars now stands at (likely more than) 204 (Tables 2 8). It goes without saying that, like any such list, this one is incomplete. Future observations would continue to add new nulling pulsars to this list, which may even exhibit hitherto unobserved characteristic features. However, the current size of nulling pulsar population is such that it allows us to draw certain broad conclusions about this sub-population of the larger class of RPP. In this work, we examine the distribution of NF and its correlation (or absence thereof) with various pulsar parameters. We also examine the general characteristics of the nulling pulsar population and revisit the connection of age with nulling behaviour. 


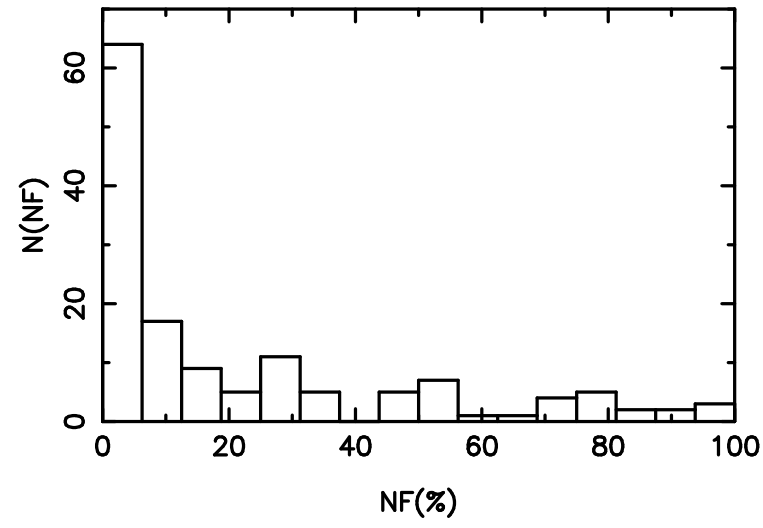

Figure 1: Histogram showing the distribution of NF, as available in the literature. Details can be found in Tables [2]-[5].

\section{Characteristics of Nulling Pulsar Population}

Only about $8 \%$ of all known radio pulsars $(\sim 2500)$ are known to exhibit nulling (Tables $2-8)$. Quite likely this fraction is much larger, as only a small number of radio pulsars are observed over long periods (or regularly) to detect nulling episodes. Also, short nulls (nulling episode lasting only for a few pulses) may not be detected in weak pulsars. 7 among these are known to belong to the class of Intermediate Pulsars. Moreover, there exist a significant number of nulling pulsars for which no estimate for NF is available (Tables 6-77). Nevertheless it is possible to draw certain broad conclusions about the population. In this context, finding a correlation of NF with an intrinsic pulsar parameter (spinperiod, characteristic age, magnetic field etc.) has been very important (Ritchings 1976; Wang, Manchester, \& Johnston 2007). Analysing 72 nulling pulsars Biggs (1992) found the spin-period $\left(\mathrm{P}_{\mathrm{s}}\right)$ to be directly proportional to NF, consistent with an earlier work by Ritchings (1976). Later, characteristic age $\left(\tau_{c}\right)$ was found to be correlated with NF (Wang, Manchester, \& Johnston 2007). Cordes \& Shannon (2008) have also reported of finding some correlation of the nulling phenomenon with small inclination angles (angle between the rotation and the magnetic axes). These observations led to the suggestion that older pulsars are harder to detect as they spend more time in their null state (Ritchings 1976) and that the phenomenon of nulling is associated with the advanced age of a pulsar.

We find, that the NF histogram (Fig.1) is suggestive of some kind of bunching at lower values of NF, and a

\begin{tabular}{|r|r|l|l|}
\hline \multirow{5}{*}{$\mathrm{NF}>40 \%$} & & $\mathrm{r}_{\mathrm{P}}$ & $\sigma$ \\
& & & \\
& $\mathrm{NF}-\mathrm{P}_{\mathrm{s}}$ & -0.403 & 0.027 \\
$\mathrm{NF}-\mathrm{B}_{\mathrm{s}}$ & -0.220 & 0.242 \\
$\mathrm{NF}-\dot{\mathrm{P}}$ & -0.119 & 0.528 \\
$\mathrm{NF}-\tau_{\mathrm{c}}$ & -0.047 & 0.807 \\
$\mathrm{NF}<40 \%$ & $\mathrm{NF}-\mathrm{DM}$ & 0.117 & 0.537 \\
& & \\
& $\mathrm{NF}-\mathrm{P}_{\mathrm{s}}$ & 0.327 & 0.0004 \\
& $\mathrm{NF}-\mathrm{B}_{\mathrm{s}}$ & 0.134 & 0.160 \\
& $\mathrm{NF}-\dot{\mathrm{P}}$ & 0.030 & 0.751 \\
& $\mathrm{NF}-\tau_{\mathrm{c}}$ & 0.100 & 0.295 \\
& $\mathrm{NF}-\mathrm{DM}$ & -0.017 & 0.861 \\
& & & \\
$\mathrm{ALL}$ & $\mathrm{NF}-\mathrm{P}_{\mathrm{s}}$ & 0.171 & 0.044 \\
& $\mathrm{NF}-\mathrm{B}_{\mathrm{s}}$ & -0.020 & 0.813 \\
$\mathrm{NF}-\dot{\mathrm{P}}$ & -0.091 & 0.283 \\
& $\mathrm{NF}-\tau_{\mathrm{c}}$ & 0.168 & 0.047 \\
$\mathrm{NF}-\mathrm{DM}$ & 0.180 & 0.033 \\
& & & \\
\hline
\end{tabular}

Table 1: Pearson's correlation coefficient $\left(\mathrm{r}_{\mathrm{P}}\right)$ for NF with various intrinsic pulsar parameters and the significance level $(\sigma)$ of the calculated value of $\mathrm{r}_{\mathrm{P}}$. $\left[\mathrm{P}_{\mathrm{s}}\right.$ - spin-period, $\mathrm{B}_{\mathrm{s}}$ - derived surface magnetic field, $\dot{\mathrm{P}}$ spin-period derivative, $\tau_{c}$ - characteristic age, DM - dispersion measure] 
likely separation of NF values at $\sim 40 \%$ (although the data size is too small to find any clear indication for two different NF populations). The general characteristics of the nulling population, as seen in Fig 2 is as follows $-$

- $-0.5 \lesssim \log P_{s} \lesssim 0.5$;

- $10^{11} \mathrm{G} \lesssim B_{s} \lesssim 10^{13} \mathrm{G}$;

- $10^{6} \mathrm{Yr} \lesssim \tau_{c} \lesssim 10^{8} \mathrm{Yr} ;$ and

- 10 pc. $\mathrm{cm}^{-3} \lesssim D M \lesssim 10^{3}$ pc.cm $\mathrm{cm}^{-3}$.

It is evident that there does not appear to be any correlation of NF with any of the intrinsic parameters as per present data. Pearson correlation coefficients (von Mises 1980) calculated to find the level of correlation of NF with various pulsars parameters, as seen in Table[1], clearly demonstrate this. This behaviour also appears to be the same for pulsars with high as well low values of NF.

From Fig 3 , it can be seen that the earlier conjecture, that nulling is predominantly experienced by old radio pulsars with relatively smaller magnetic fields, appears to be ruled out by the current population.

Interestingly, the nature of the distribution of the intrinsic parameters appear to be very different for pulsars exhibiting high NF compared to those having low values of NF. A nominal Kolmogorov-Smirnov (KS) test (von Mises 1980) on the spin-period of nulling pulsars with higher and lower values of NF, yields $\mathrm{P}_{\mathrm{KS}}$ $=0.002$ and $D_{K S}=0.322$, rejecting the null hypothesis that these two populations have the same underlying distribution. [Here $\mathrm{P}_{\mathrm{KS}}$ indicates the probability that the two distributions are inherently similar (identical), whereas $D_{K S}$ is the maximum value of the absolute difference between the two distributions.] This is also evident from the fractional plot of period distributions shown in Fig 4

\section{Pulsar Death-Lines}

Clearly, the nature of the emission mechanism must have a bearing on the nulling behaviour, whether or not nulling is directly related to the age of a pulsar. As mentioned earlier, Ritchings (1976) undertook the first comprehensive study of nulling pulsars and suggested that the time interval between regular bursts of pulse emission increases with age, eventually leading to pulsar 'death'. This study explicitly defined, for the very first time, a cut-off line for pulsar emission.
This can be thought of as the precursor of more formal 'death-line's to be developed afterwards. Later, Zhang, Gil, \& Dyks (2007) also suggested that nulling pulsars are likely to be very close to the death line, being active only when favourable conditions prevail.

Irrespective of the underlying mechanism, copious pair production in the magnetosphere is understood to be the basic requirement for pulsar emission. Such pair production gives rise to a dense plasma that can then allow the growth of a number of coherent instabilities and generate highly relativistic secondary pairs which then produce the radio band emission (see Mitra, Gil, \& Melikidze 2009, Melrose 2017 and references therein for details of and recent progresses made in the area of pulsar emission physics).

Pulsars 'switch off' when conditions for pair production fail to be met. Depending on the specific model, radio pulsar 'death line' is defined to be a relation between $\mathrm{P}_{\mathrm{s}} \& \dot{\mathrm{P}}$ (period derivative) or $\mathrm{P}_{\mathrm{s}} \& \mathrm{~B}_{\mathrm{s}}$ beyond which the process of pair-production ceases and a pulsar stops emitting. A number of theoretical models, consequently a variety of death-lines have been proposed to explain the present crop of pulsars. All of these require some degree of anomalous field configuration (higher multipole components or an offset dipole) to interpret the data in its entirety. Some of the most representative death-lines, based upon different models of emission mechanism, are described below.

In the following equations - $B_{\mathrm{p}}$ is the dipolar field, $B_{\mathrm{s}}$ is the surface field, $r_{\mathrm{c}}$ is the radius of curvature for the magnetic field, $h$ is the thickness of the polar cap gap, $R$ is the stellar radius and $\Omega$ is the stellar spin frequency. The value of inclination angle chosen for $\mathbf{5 b}$ corresponds to that for Geminga.

\section{A. Chen \& Ruderman (1993) :}

I. Polar Cap Model : Pair production $\left(\gamma+B \rightarrow e^{-}+\right.$ $e^{+}$) predominantly happens near the polar cap of the neutron star (Ruderman \& Sutherland 1975).

01. Central Dipole, with $B_{\mathrm{s}}=B_{\mathrm{p}}, r_{\mathrm{c}}=(R c / \Omega)^{1 / 2}$ -

$4 \log B-7.5 \log P=49.3$,

01a. Dipole, off-centre by a distance $d$ -

$4 \log B-7.5 \log P=49.3-2.5 \log [R /(R-d)]$,

02. Very curved field lines, with $r_{\mathrm{c}} \sim R, B_{\mathrm{s}}=B_{\mathrm{p}}$ -

$4 \log B-6.5 \log P=45.7$,

03. Very curved field lines, with $r_{\mathrm{c}} \sim R, B_{\mathrm{s}}=2 \times 10^{13} \mathrm{G}$, $h \sim\left(B_{\mathrm{p}} / B_{\mathrm{s}}\right)^{1 / 2} R(R \Omega / c)^{1 / 2}$ at polar cap -

$7 \log B-13 \log P=78$, 

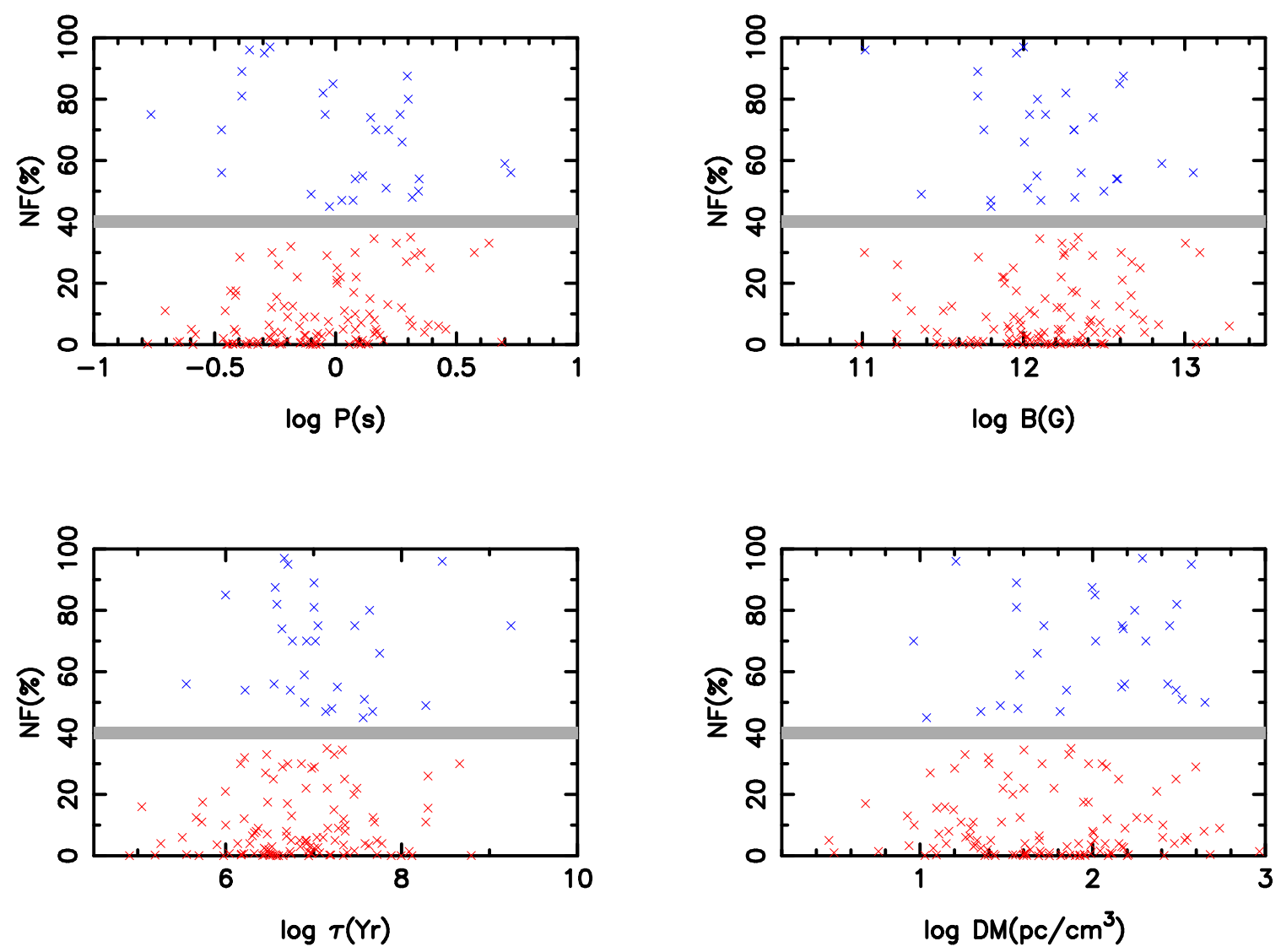

Figure 2: Variation of the nulling fraction $(\mathrm{NF})$ against spin-period $\left(\mathrm{P}_{\mathrm{s}}\right)$, surface magnetic field $\left(\mathrm{B}_{\mathrm{s}}\right)$, characteristic age $\left(\tau_{\mathrm{c}}\right)$ and dispersion measure (DM) of pulsars. The red points correspond to pulsars with low $\mathrm{NF}(<40 \%)$ and the blue points to pulsars with larger values of NF. The horizontal grey band highlights the apparent gap in NF values around $40 \%$. 

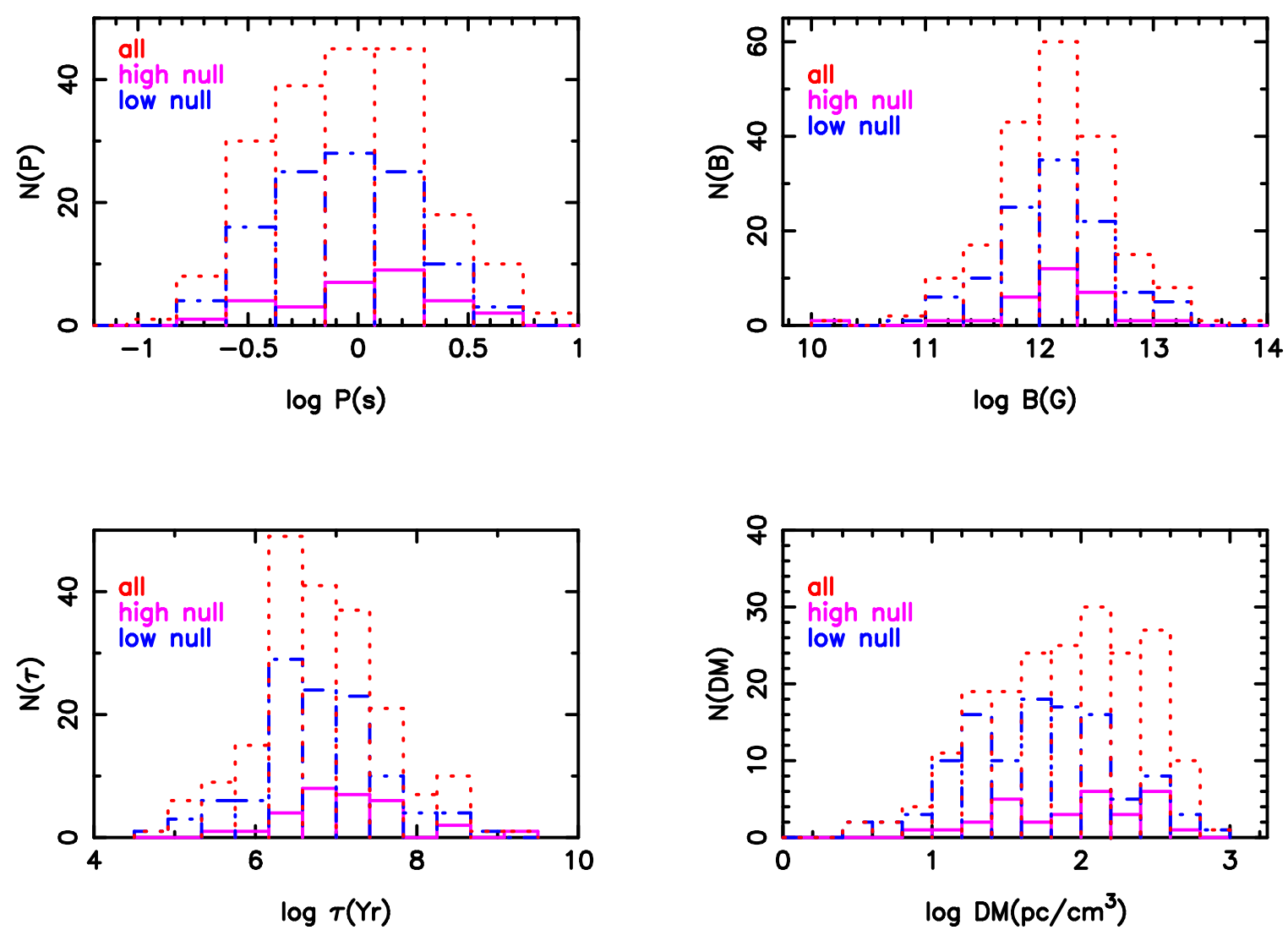

Figure 3: Distribution of characteristic pulsar parameters for pulsars with high null ( $\mathrm{NF}<40 \%)$, with low null (NF $>40 \%$ ), and all of the nulling pulsars. It is to be noted that the histogram for all nulling pulsars also include pulsars without any estimate for NF and the intermittent pulsars. 


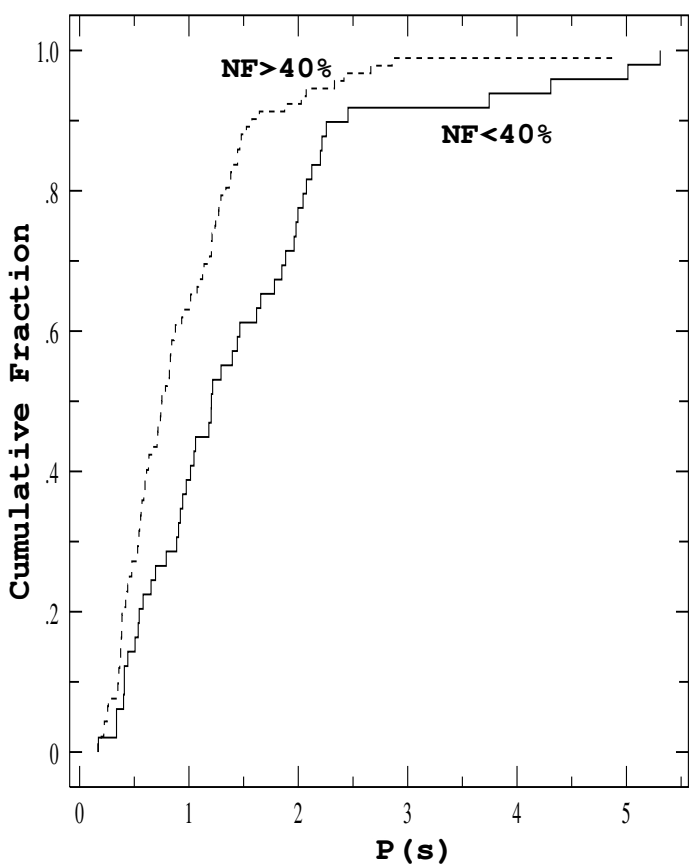

Figure 4: Cumulative fraction plot for the $\mathrm{P}_{\mathrm{s}}$ distribution of pulsars with low $(<40 \%)$ and high $(>40 \%)$ values of NF.

04a, 04b. Extremely twisted field lines, with $r_{\mathrm{c}} \sim R$ $4 \log B-6 \log P=43.8$ or 31.3 ,

(Whichever constant produces larger B in the equation above to ensure $E_{\mathrm{p}}>2 m_{e} c^{2}$.)

II. Outer Magnetospheric Model : Pair production happens in the outer magnetosphere via inverse Compton scattering, curvature radiation or synchrotron radiation etc.

05a, 05b. Aligned/Non-Aligned Dipole -

$5 \log B-12 \log P=72$ or 69.5 .

B. Zhang, Harding, \& Muslimov (2000) : In each of the pair of equations below (depicted by the sets 06a06b, 07a-07b, 08a-08b, 09a-09b), the first one corresponds to a dipole configuration and the second one corresponds to a multipolar configuration with $B_{\mathrm{s}} \sim B_{\mathrm{p}}$ and $r_{\mathrm{c}} \sim R$. Furthermore, $r_{\mathrm{c} 6}$ is $r_{\mathrm{c}}$ in units of $10^{6} \mathrm{~cm}$.

I. Vacuum Gap Model : Pair production happens via formation of a vacuum gap close to the polar cap.

A. Curvature Radiation -

06a. $\log \dot{\mathrm{P}}=11 / 4 \log P-14.62$,

06b. $\log \dot{\mathrm{P}}=9 / 4 \log P-16.58+\log r_{\mathrm{c} 6}$,
B. Inverse Compton Scattering -
07a. $\log \dot{\mathrm{P}}=2 / 11 \log P-13.07$,

07b. $\log \dot{\mathrm{P}}=-2 / 11 \log P-14.50+8 / 11 \log r_{\mathrm{c} 6}$,

II. Space-Charge Limited Flow Model : If charged particles can be freely pulled out of the neutron star surface, a space-charged limited flow is generated. Mechanisms similar to those above then work to generate secondary/tertiary pairs.

A. Curvature radiation -

08a. $\log \dot{\mathrm{P}}=5 / 2 \log P-14.56$,

08b. $\log \dot{\mathrm{P}}=2 \log P-16.52+\log r_{\mathrm{c} 6}$,

B. Inverse Compton Scattering -

09a. $\log \dot{\mathrm{P}}=-3 / 11 \log P-15.36$,

09b. $\log \dot{\mathrm{P}}=-7 / 11 \log P-16.79+8 / 11 \log r_{\mathrm{c} 6}$.

All the death-lines discussed above have been indicated in the top panel of Fig. [5], in the backdrop of the known radio pulsars in $\mathrm{P}_{\mathrm{s}}-\mathrm{B}_{\mathrm{s}}$ plane. It should be noted that while the death lines are defined in terms of the magnetic field by Chen \& Ruderman (1993), they are defined using the derivative of spin-period $(\dot{\mathrm{P}})$ by Zhang, Harding, \& Muslimov (2000). However, the magnetic field is not a measured quantity. An estimate, only for the dipolar component, is obtained from the measured quantities $\mathrm{P}_{\mathrm{s}}$ and $\dot{\mathrm{P}}$ through the following relation (Manchester \& Taylor 1977) -

$B_{\mathrm{p}} \simeq 3.2 \times 10^{19}\left(\frac{P_{s}}{s}\right)^{\frac{1}{2}}\left(\frac{\dot{\mathrm{P}}}{s s^{-1}}\right)^{\frac{1}{2}} \mathrm{G}$.

In Fig. [5], this measure of the magnetic field is used for known pulsars. The death-line equations, given in terms of $\mathrm{P}_{\mathrm{s}}$ and $\dot{\mathrm{P}}$ by Zhang, Harding, \& Muslimov $(2000)$, are also plotted in the $\mathrm{P}_{\mathrm{s}}-\mathrm{B}_{\mathrm{s}}$ plane using the same measure. Therefore, any conclusion drawn for this set of death-line equations do not suffer from an ambiguity regarding the measure of the magnetic field (between the dipolar and the true surface field). However, that is not correct in case of the death-lines defined by Chen \& Ruderman (1993), which suffer from this 
ambiguity. It is clear that the death-lines $\mathbf{7 a - 7 b}, \mathbf{9 a}$ 9b are not very useful in constraining the radio pulsar population. In particular, they completely fail to accommodate the millisecond pulsars. Even if one questions whether the same death-line works for both the ordinary and millisecond pulsars, because these equations also preclude a significant number of relatively low field pulsars, we shall not consider them hereafter. On the other hand, the death-line depicted by $\mathbf{4 b}$ is far too deep into the 'graveyard' to be of much use for the current population of pulsars. Perhaps the newly discovered long-period pulsars (some of which have been indicated by red stars in the bottom panel of Fig. [5]) are likely to be constrained by this equation. Among the rest, 1, 6a and 2, $\mathbf{6 b}$ are pairwise coincident (almost); while $\mathbf{2}, 3$ and 4 envelope somewhat similar regions. $(\mathbf{8 a}, 8 \mathbf{b}$ are, more or less, coincident with $\mathbf{6 a}, \mathbf{6 b}$ and therefore not shown in the figure.)

In the bottom panel of Fig. [5] the nulling pulsars (along with intermediate pulsars) are shown along with a relevant subset of death-lines. A number of (non-nulling) pulsars have been also been identified for their importance in the context of death-lines. For example, despite the wide variety of models and the large number of possible death-lines described above, it was necessary to invoke higher multipoles, many orders of magnitude stronger than the dipole, at the the surface to accommodate the $8.5 \mathrm{~s}$ pulsar J2144-3933 Gil \& Mitra (2001). Other pulsars, like J1232-3933 (Jacoby et al. 2009), J1333-4449 (Jacoby et al. 2009) or J2123+5454 (Stovall et al. 2014) may also have similar explanations for them to work beyond the death-line $4 \mathbf{a}$. It is to be noted that J0250+5854 (Tan et al. 2018), the famous slow pulsar $\left(\mathrm{P}_{\mathrm{s}}=23.5 \mathrm{~s}\right)$, is actually within the allowed-zone, as far as death-lines are concerned.

It is likely that more than one emission mechanism could be responsible for radio pulsars activity (Chen \& Ruderman 1993). It is then plausible that different death-lines are appropriate for pulsars in which different mechanisms are responsible for the emission. Though, at present, there is no clear understanding of this. However, when the population of nulling pulsars are marked out in the $\mathrm{P}_{\mathrm{s}}-\mathrm{B}_{\mathrm{s}}$ plane, certain remarkable things are noticed. It can be seen from the bottom panel of Fig. [5] that there are almost no nulling pulsar above the death-line 5b (definitely none above 5a). Now, $\mathbf{5 a}, \mathbf{5 b}$ correspond to pure dipolar field configurations (aligned or non-aligned with the rotation axis) in an outer magnetospheric model. Given the current understanding of pulsar emission process, this may mean that the nulling pulsars likely do not possess purely dipolar field configurations where emission originates in the outer magnetosphere. On the other hand, the nulling pulsars appear to be bounded below by death-line $\mathbf{2}$, which corresponds to a polar cap emission model with very curved field lines (curvature radius $\sim$ stellar radius). Taken together, it is suggestive of the conclusion that the pulsars for which the emission is predominantly from the polar cap and the magnetic field is extremely curved are likely to experience nulling episodes.

It has been suggested that in some pulsars, the magnetosphere may occasionally switch ('mode change') between different states with different geometries or/and different distributions of currents (Timokhin 2010). These states have different spin-down rates and emission beams; and some of the states do not (or apparently not) have any radio emission. In case of the intermittent pulsar, B1931+24, it has been clearly seen that $\dot{\mathrm{P}}$ significantly differs from the off-state (nulling phase) to the on-state (active phase). It has been argued that, in the off-phase, the open field lines above the magnetic pole become depleted of charged particles and the rotational slow down happens purely due to the magnetic dipole radiation. On the other hand, in the onphase, an additional slow-down torque is provided by the out-flowing plasma (Kramer et al. 2006a). Therefore, an estimate of the dipolar magnetic field obtained from measurements made during the active phase is always an overestimate. On the other hand, Young et al. (2012) has reported to have observed no change in the spin-down rate for the pulsar $\mathrm{B} 0823+26$ between the off-state and the on-state. This implies that there would be no overestimate of the dipolar field for this pulsar. Given this, it is difficult to gauge whether the reported values of $\dot{\mathrm{P}}$ and hence that of the dipolar field is an overestimate or not. However, even with a $10 \%$ overestimate (assumed for all the nulling pulsars), we find that our conclusions drawn above remain unchanged.

It is clear from the bottom-panel of Fig. [5] that quite a large number of pulsars are active beyond the deathline 2 , but are bounded by the death-line 4a which again corresponds to polar-cap emission but the magnetic field configurations for this case are extremely twisted. Because the pulsars in this region (between death-lines $\mathbf{2}$ and $\mathbf{4 a}$ ) are slow objects with no apparent significance they have mostly not been studied in detail. To our knowledge, there does not exist any study that specifically investigates the nulling behaviour of pulsars in this region. However, it is quite clear that if these objects are carefully monitored, for the presence of any nulling episodes, we would be able to gauge the validity of the conclusion above. With this in mind, we are initiating such a program, of targeted observation of slow pulsars between the death-lines $\mathbf{2}$ and $\mathbf{4 a}$, with the Giant Meterwave Radio Telescope (Konar, Roy, \& Bhattacharyaa 2019). 

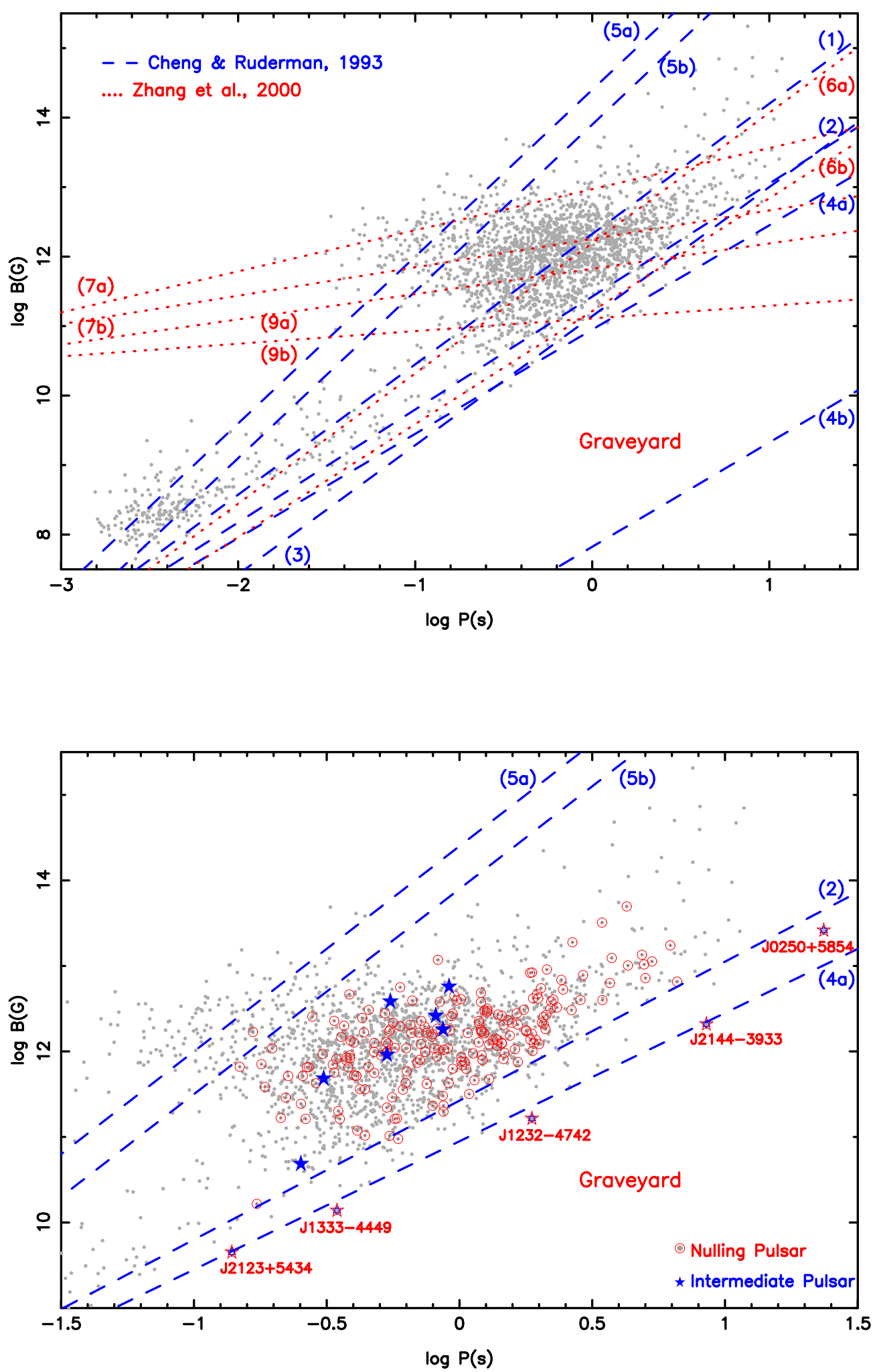

Figure 5: Observed radio pulsars and theoretical death-lines in the $\mathrm{P}_{\mathrm{s}}-\mathrm{B}_{\mathrm{s}}$ plane. Top Panel - The death lines have been marked according to their numbering in the text. Bottom Panel - Nulling pulsars and intermediate pulsars have been highlighted with a small subset of death-lines. A number of pulsars have been specially identified (red open star) which appear to be functioning beyond the least stringent death line. The data for the known pulsars have been obtained from the ATNF pulsar catalog http://www.atnf.csiro.au/research/pulsar/psrcat/(Manchester et al., 2005). 


\section{Summary}

About $8 \%$ of all known radio pulsars have been observed to exhibit nulling. In this work, we have considered NF to be the marker (for want of any other characteristic parameter which has been estimated for a significant number of nulling pulsars) for a pulsar's nulling behaviour and have looked at the nature of its distribution. We have also considered the general characteristics (in terms of intrinsic pulsar parameters) of this sub-population of radio pulsars. The conclusions drawn are summarised as follows -

1. There appears to be a gap in the estimated value of nulling fraction around $40 \%$, separating pulsars into two populations exhibiting higher and lower values of NF. However, this should be taken with a bit of caution, as inaccurate estimates of NF and inadequate study of pulsars with NF near $40 \%$ could contribute to this bias. On the other hand, the error bars, even though these could be quite large on occasion (tables [2 - 5]), do not appear likely to smudge out the gap.

2. The number of pulsars with a lower value $(<40 \%)$ of NF appear to be far more in comparison to the ones with a higher value of $\mathrm{NF}$ (Fig.[1]). Once again, this could simply be an artifact of observational bias. For example, pulsars with very high NF are quite likely to be entirely missed by rapid pulsar surveys where other pulsars with zero (or small) nulling fractions are detected easily.

3. The distributions of the intrinsic pulsar parameters $\left(\mathrm{P}_{\mathrm{s}}, \dot{\mathrm{P}}, \mathrm{B}_{\mathrm{s}}, \tau_{\mathrm{c}}\right.$, DM etc.) are statistically different in these two populations of pulsars with high and low values of NF.

4. There is no evidence of any correlation of NF with any of the intrinsic pulsar parameters as per present data. This behaviour is similar for pulsars with high as well as low values of NF.

5. The most interesting conclusion of our study is regarding the nature of the nulling pulsars. It appears likely that pulsars, for which the emission is predominantly from the polar cap and have extremely curved magnetic fields, preferentially experience nulling episodes. If borne out by future observations, this would pave the way for a theory of nulling which has so far eluded us.

6. Regular and targeted monitoring of pulsars in the region close to and bounded by the death-lines 2 and $\mathbf{4 a}$ is therefore of great importance. As mentioned earlier, we are initiating a study with this goal.

\section{Acknowledgment}

Most of this work had been carried out when SK was supported by a grant (SR/WOS-A/PM-1038/2014) from DST, Government of India and UD was supported through the 'Indian Academies' Summer Research Fellowship Programme (2017)'. Work done by Devansh Agarwal (also as a part of another summer project) has been useful in sorting out certain preliminary issues. SK would also like to thank Avinash Deshpande, Yashwant Gupta, Vishal Gajjar and Bhal Chandra Joshi for helpful discussions. We also thank the anonymous referee for helping to improve the clarity of the manuscript significantly.

\section{References}

Backer D. C., 1970, Nature, 228, 42

Basu R., Mitra D., 2018, MNRAS, 476, 1345

Basu R., Mitra D., Melikidze G. I., 2017, ApJ, 846, 109

Bhattacharyya B., Gupta Y., Gil J., 2010, MNRAS, 408, 407

Biggs J. D., 1992, ApJ, 394, 574

Brinkman C., Freire P. C. C., Rankin J., Stovall K., 2018, MNRAS, 474, 2012

Burke-Spolaor S., Bailes M., 2010, MNRAS, 402, 855

Burke-Spolaor S. et al., 2011, MNRAS, 416, 2465

Burke-Spolaor S. et al., 2012, MNRAS, 423, 1351

Camilo F., Ransom S. M., Chatterjee S., Johnston S., Demorest P., 2012, ApJ, 746, 63

Chen K., Ruderman M., 1993, ApJ, 402, 264

Cordes J. M., Shannon R. M., 2008, ApJ, 682, 1152

Crawford F., Lorimer D., Ridley J., Madden J., 2013, in American Astronomical Society Meeting Abstracts, Vol. 221, American Astronomical Society Meeting Abstracts \#221, p. 412.04

Deneva J. S. et al., 2016, ApJ, 821, 10

Deneva J. S., Stovall K., McLaughlin M. A., Bates S. D., Freire P. C. C., Martinez J. G., Jenet F., Bagchi M., 2013, ApJ, 775, 51

Deshpande A. A., Rankin J. M., 2001, MNRAS, 322, 438

Durdin J. M., Large M. I., Little A. G., Manchester R. N., Lyne A. G., Taylor J. H., 1979, MNRAS, 186, 39P

Dyks J., Zhang B., Gil J., 2005, ApJ, 626, L45

Esamdin A., Lyne A. G., Graham-Smith F., Kramer M., Manchester R. N., Wu X., 2005, MNRAS, 356, 59

Faulkner A. J. et al., 2004, MNRAS, 355, 147 
Filippenko A. V., Radhakrishnan V., 1982, ApJ, 263, 828 Gajjar V., 2017, arXiv:1706.05407

Gajjar V., Joshi B. C., Kramer M., 2012, MNRAS, 424, 1197

Gajjar V., Joshi B. C., Kramer M., Karuppusamy R., Smits R., 2014, ApJ, 797, 18

Gajjar V., Yuan J. P., Yuen R., Wen Z. G., Liu Z. Y., Wang N., 2017, ApJ, 850, 173

Gil J., Mitra D., 2001, ApJ, 550, 383

Herfindal J. L., Rankin J. M., 2007, MNRAS, 380, 430

Herfindal J. L., Rankin J. M., 2009, MNRAS, 393, 1391

Jacoby B. A., Bailes M., Ord S. M., Edwards R. T., Kulkarni S. R., 2009, ApJ, 699, 2009

Johnston S., Lyne A. G., Manchester R. N., Kniffen D. A., D’Amico N., Lim J., Ashworth M., 1992, MNRAS, 255, 401

Joshi B. C. et al., 2009, MNRAS, 398, 943

Kaspi V. M., 2010, Proceedings of the National Academy of Science, 107, 7147

Konar S., 2013, in Astronomical Society of India Conference Series, Vol. 8, Das S., Nandi A., Chattopadhyay I., ed, Astronomical Society of India Conference Series, p. 89

Konar S., 2017, Journal of Astrophysics and Astronomy, 38,47

Konar S., 2019, in prep

Konar S. et al., 2016, Journal of Astrophysics and Astronomy, 37, 36

Konar S., Roy J., Bhattacharyaa B., 2019, in prep

Kramer M., Lyne A. G., O’Brien J. T., Jordan C. A., Lorimer D. R., 2006a, Science, 312, 549

Kramer M. et al., 2006b, Science, 314, 97

Li J., Esamdin A., Manchester R. N., Qian M. F., Niu H. B., 2012, MNRAS, 425, 1294

Lorimer D. R., Camilo F., Xilouris K. M., 2002, AJ, 123, 1750

Lorimer D. R., Lyne A. G., McLaughlin M. A., Kramer M.,

Pavlov G. G., Chang C., 2012, ApJ, 758, 141

Lynch R. S. et al., 2013, ApJ, 763, 81

Lyne A., Hobbs G., Kramer M., Stairs I., Stappers B., 2010,

Science, 329, 408

Lyne A. G., McLaughlin M. A., Keane E. F., Kramer M., Espinoza C. M., Stappers B. W., Palliyaguru N. T., Miller J., 2009, MNRAS, 400, 1439

Lyne A. G. et al., 2017, ApJ, 834, 72

Manchester R. N., Hobbs G. B., Teoh A., Hobbs M., 2005, VizieR Online Data Catalog, 7245, 0

Manchester R. N., Taylor J. H., 1977, Pulsars. W. H. Freeman, San Francisco, San Francisco, p. 36

McLaughlin M. A. et al., 2006, Nature, 439, 817

Melrose D. B., 2017, Reviews of Modern Plasma Physics, 1,5

Meyers B. W. et al., 2018, ApJ, 869, 134

Mitra D., Gil J., Melikidze G. I., 2009, ApJ, 696, L141

Naidu A., Joshi B. C., Manoharan P. K., KrishnaKumar M. A., 2017, A\&A, 604, A45
Naidu A., Joshi B. C., Manoharan P. K., Krishnakumar M. A., 2018, MNRAS, 475, 2375

O'Brien J. T., Kramer M., Lyne A. G., Lorimer D. R., Jordan C. A., 2006, Chinese Journal of Astronomy and Astrophysics Supplement, 6, 4

Rajwade K., Gupta Y., Kumar U., Arjunwadkar M., 2014, in Astronomical Society of India Conference Series, Vol. 13, Astronomical Society of India Conference Series, p. 73

Rankin J. M., 1986, ApJ, 301, 901

Rankin J. M., Rathnasree N., 1995, Journal of Astrophysics and Astronomy, 16, 327

Rankin J. M., Wright G. A. E., 2007, MNRAS, 379, 507

Rankin J. M., Wright G. A. E., 2008, MNRAS, 385, 1923

Rankin J. M., Wright G. A. E., Brown A. M., 2013, MNRAS, 433, 445

Redman S. L., Rankin J. M., 2009, MNRAS, 395, 1529

Ritchings R. T., 1976, MNRAS, 176, 249

Rosen R. et al., 2013, ApJ, 768, 85

Ruderman M. A., Sutherland P. G., 1975, ApJ, 196, 51

Stovall K. et al., 2014, ApJ, 791, 67

Surnis M. P., Joshi B. C., McLaughlin M. A., Gajjar V., 2013, in IAU Symposium, Vol. 291, IAU Symposium, p. 508

Tan C. M. et al., 2018, ApJ, 866, 54

Timokhin A. N., 2010, MNRAS, 408, L41

Vivekanand M., 1995, MNRAS, 274, 785

von Mises R., 1980, Mathematical Theory of Probability and Statistics. Academic Press, New York

Wang N., Manchester R. N., Johnston S., 2007, MNRAS, 377,1383

Weisberg J. M., Armstrong B. K., Backus P. R., Cordes J. M., Boriakoff V., Ferguson D. C., 1986, AJ, 92, 621

Yang A., Han J., Wang N., 2014, Science China Physics, Mechanics, and Astronomy, 57, 1600

Young N. J., Stappers B. W., Weltevrede P., Lyne A. G., Kramer M., 2012, MNRAS, 427, 114

Young N. J., Weltevrede P., Stappers B. W., Lyne A. G., Kramer M., 2014, MNRAS, 442, 2519

Young N. J., Weltevrede P., Stappers B. W., Lyne A. G., Kramer M., 2015, MNRAS, 449, 1495

Zhang B., Gil J., Dyks J., 2007, MNRAS, 374, 1103

Zhang B., Harding A. K., Muslimov A. G., 2000, ApJ, 531, L135

\section{Appendix A. Nulling Pulsar Parameters}


Table 2: Characteristic parameters - spin-period $\left(\mathrm{P}_{\mathrm{s}}\right)$, surface magnetic field $\left(\mathrm{B}_{\mathrm{s}}\right)$ and nulling fraction (NF) of known nulling pulsars. The $\mathrm{P}_{\mathrm{s}}$ and $\mathrm{B}_{\mathrm{s}}$ values are taken from the ATNF database (http://www.atnf.csiro.au/research/pulsar/psrcat/) while NF values have been indicated with appropriate references, inclusive of cases where different estimates have been reported by different groups.

\begin{tabular}{|c|c|c|c|c|c|c|}
\hline & PR'S Name & J-Name & $\begin{array}{l}P_{s} \\
(s)\end{array}$ & $\begin{array}{l}B_{s} \\
(G)\end{array}$ & $\begin{array}{l}\mathrm{NF} \\
(\%)\end{array}$ & References \\
\hline 1 & B0031-07 & J0034-0721 & 0.9429 & $6.28 \times 10^{11}$ & $44.0 \pm 1.0$ & Gajjar 2017 \\
\hline 2 & B $0045+33$ & $\mathrm{~J} 0048+3412$ & 1.2171 & $1.71 \times 10^{12}$ & $21.0 \pm 1.0$ & Redman \& Rankin 2009 \\
\hline 3 & B0148-06 & J0151-0635 & 1.4647 & $8.15 \times 10^{11}$ & $\leq 5.0$ & Biggs 1992 \\
\hline 4 & B0149-16 & J0152-1637 & 0.8327 & $1.05 \times 10^{12}$ & $\leq 2.5$ & Vivekanand 1995 \\
\hline 5 & В0301+19 & J0304+1932 & 1.3876 & $1.36 \times 10^{12}$ & 10.0 & Rankin 1986 \\
\hline 6 & B $0329+54$ & $\mathrm{~J} 0332+5434$ & 0.7145 & $1.22 \times 10^{12}$ & $\leq 0.25$ & Ritchings 1976 \\
\hline 7 & B0450-18 & J0452-1759 & 0.5489 & $1.80 \times 10^{12}$ & $\leq 0.5$ & Ritchings 1976 \\
\hline 8 & $\mathrm{~J} 0458-0505$ & J0458-0505 & 1.8835 & $1.01 \times 10^{12}$ & $63.0 \pm 3.0$ & Lynch et al. 2013 \\
\hline 9 & $\mathrm{~B} 0523+11$ & $\mathrm{~J} 0525+1115$ & 0.3544 & $1.63 \times 10^{11}$ & $\leq 0.06$ & Weisberg et al. 1986 \\
\hline 10 & $\mathrm{~B} 0525+21$ & $\mathrm{~J} 0528+2200$ & 3.7455 & $1.24 \times 10^{13}$ & $25.0 \pm 5.0$ & Ritchings 1976 \\
\hline 11 & B0529-66 & J0529-6652 & 0.9757 & $3.94 \times 10^{12}$ & $83.5 \pm 1.5$ & Crawford et al. 2013 \\
\hline 12 & B $0626+24$ & $\mathrm{~J} 0629+2415$ & 0.4766 & $9.87 \times 10^{11}$ & $\leq 0.02$ & Weisberg et al. 1986 \\
\hline 13 & B0628-28 & J0630-2834 & 1.2444 & $3.01 \times 10^{12}$ & $\leq 0.3$ & Biggs 1992 \\
\hline 14 & B $0656+14$ & J0659+1414 & 0.3849 & $4.66 \times 10^{12}$ & $\overline{12.0 \pm 4.0}$ & Weisberg et al. 1986 \\
\hline 15 & В0736-40 & J0738-4042 & 0.3749 & $7.88 \times 10^{11}$ & $\leq 0.4$ & Biggs 1992 \\
\hline 16 & B0740-28 & $\mathrm{J} 0742-2822$ & 0.1668 & $1.69 \times 10^{12}$ & $\leq 0.2$ & Biggs 1992 \\
\hline 17 & B $0751+32$ & $\mathrm{~J} 0754+3231$ & 1.4423 & $1.26 \times 10^{12}$ & $\overline{3} 4.0 \pm 0.5$ & Weisberg et al. 1986 \\
\hline 18 & B $0809+74$ & J0814+7429 & 1.2922 & $4.72 \times 10^{11}$ & $\leq 5.0$ & Ritchings 1976 \\
\hline 19 & B0818-13 & J0820-1350 & 1.2381 & $1.63 \times 10^{12}$ & $1.5 \pm 0.25$ & Ritchings 1976 \\
\hline 20 & B0818-41 & J0820-4114 & 0.5454 & $1.03 \times 10^{11}$ & 30.0 & Bhattacharyya, Gupta, \& Gil 2010 \\
\hline 21 & B0820+02 & J0823+0159 & 0.8649 & $3.04 \times 10^{11}$ & $\leq 0.06$ & Weisberg et al. 1986 \\
\hline 22 & $\mathrm{~B} 0823+26$ & $\mathrm{~J} 0826+2637$ & 0.5307 & $9.64 \times 10^{11}$ & $6.4 \pm 0.8$ & Rankin \& Rathnasree 1995 \\
\hline 23 & B0826-34 & J0828-3417 & 1.8489 & $1.37 \times 10^{12}$ & $75.0 \pm 35.0$ & Durdin et al. 1979 \\
\hline 24 & B0833-45 & J0835-4510 & 0.0893 & $3.38 \times 10^{12}$ & $\leq 0.0008$ & Biggs 1992 \\
\hline 25 & B0834+06 & $\mathrm{J} 0837+0610$ & 1.2738 & $2.98 \times 10^{12}$ & $\overline{7} .1 \pm 0.1$ & Ritchings 1976 \\
\hline 26 & B0835-41 & J0837-4135 & 0.7516 & $1.65 \times 10^{12}$ & $1.7 \pm 1.2$ & Gajjar 2017 \\
\hline \multirow[t]{2}{*}{27} & B0906-17 & J0908-1739 & 0.4016 & $5.25 \times 10^{11}$ & $26.8 \pm 1.7$ & Basu, Mitra, \& Melikidze 2017 \\
\hline & & & & $5.25 \times 10^{11}$ & $25.7 \pm 1.3$ & Basu, Mitra, \& Melikidze 2017 \\
\hline 28 & В0919+06 & $\mathrm{J} 0922+0638$ & 0.4306 & $2.46 \times 10^{12}$ & $\leq 0.05$ & Weisberg et al. 1986 \\
\hline 29 & B0932-52 & J0934-5249 & 1.4448 & $2.62 \times 10^{12}$ & $5.0 \pm 3.0$ & Naidu et al. 2017 \\
\hline 30 & B0940-55 & J0942-5552 & 0.6644 & $3.94 \times 10^{12}$ & $\leq 12.5$ & Biggs 1992 \\
\hline 31 & B $0940+16$ & J0943+1631 & 1.0874 & $3.18 \times 10^{11}$ & $8.0 \pm 3.0$ & Weisberg et al. 1986 \\
\hline 32 & B0942-13 & J0944-1354 & 0.5703 & $1.63 \times 10^{11}$ & $\begin{array}{l}14.4 \pm 0.9 \\
\leq 7.0\end{array}$ & \begin{tabular}{|l|} 
Basu, Mitra, \& Melikidze 2017 \\
Vivekanand 1995
\end{tabular} \\
\hline 33 & B0950+08 & J0953+0755 & 0.2531 & $2.44 \times 10^{11}$ & $\leq 5.0$ & Ritchings 1976 \\
\hline \multirow[t]{2}{*}{34} & $\mathrm{~J} 1049-5833$ & $\mathrm{~J} 1049-5833$ & 2.2023 & $3.15 \times 10^{12}$ & $47.0 \pm 3.0$ & Wang, Manchester, \& Johnston 2007 \\
\hline & & & & & $47.0 \pm 3.0$ & Yang, Han, \& Wang 2014 \\
\hline 35 & B1055-52 & $\mathrm{J} 1057-5226$ & 0.1971 & $1.09 \times 10^{12}$ & $\leq 11.0$ & Biggs 1992 \\
\hline 36 & $\mathrm{~B} 1112+50$ & $\mathrm{~J} 1115+5030$ & 1.6564 & $2.06 \times 10^{12}$ & $64.0 \pm 6.0$ & Gajjar 2017 \\
\hline 37 & B1114-41 & $\mathrm{J} 1116-4122$ & 0.9432 & $2.77 \times 10^{12}$ & $3.3 \pm 0.5$ & Basu, Mitra, \& Melikidze 2017 \\
\hline & $\mathrm{B} 1133+16$ & $\mathrm{~J} 1136+1551$ & 1.1879 & $2.13 \times 10^{12}$ & $15.0 \pm 2.5$ & Ritchings 1976 \\
\hline \multirow[t]{2}{*}{39} & $\mathrm{~B} 1237+25$ & $\mathrm{~J} 1239+2453$ & 1.3824 & $1.17 \times 10^{12}$ & $6.0 \pm 2.5$ & Ritchings 1976 \\
\hline & & & & & $7.0 \pm 3.0$ & Naidu et al. 2017 \\
\hline 40 & B1240-64 & $\mathrm{J} 1243-6423$ & 0.3885 & $1.34 \times 10^{12}$ & $\leq 4.0$ & Biggs 1992 \\
\hline
\end{tabular}


Table 3: Continuation of Table 2

\begin{tabular}{|c|c|c|c|c|c|c|}
\hline & PSR Name & J-Name & $\begin{array}{l}P_{s} \\
(s)\end{array}$ & $\begin{array}{l}B_{s} \\
(G)\end{array}$ & $\begin{array}{l}\mathrm{NF} \\
(\%)\end{array}$ & References \\
\hline 41 & B1322-66 & J1326-6700 & 0.5430 & $1.72 \times 10^{12}$ & $9.1 \pm 3.0$ & Wang, Manchester, \& Johnston 2007 \\
\hline 42 & B1325-49 & J1328-4921 & 1.4787 & $9.61 \times 10^{11}$ & 4.0 & Basu, Mitra, \& Melikidze 2017 \\
\hline 43 & B1358-63 & J1401-6357 & 0.8428 & $3.80 \times 10^{12}$ & $1.6 \pm 2.0$ & Wang, Manchester, \& Johnston 2007 \\
\hline 44 & B1426-66 & J1430-6623 & 0.7854 & $1.49 \times 10^{12}$ & $\leq 0.05$ & Biggs 1992 \\
\hline 45 & B1451-68 & J1456-6843 & 0.2634 & $1.63 \times 10^{11}$ & $\leq 3.3$ & Biggs 1992 \\
\hline 46 & $\mathrm{~J} 1502-5653$ & $\mathrm{~J} 1502-5653$ & 0.5355 & $9.99 \times 10^{11}$ & $93.0 \pm 4.0$ & Wang, Manchester, \& Johnston 2007 \\
\hline 47 & B1508+55 & $\mathrm{J} 1509+5531$ & 0.7397 & $1.95 \times 10^{12}$ & $7.0 \pm 2.0$ & Naidu et al. 2017 \\
\hline 48 & J1525-5417 & $\mathrm{J} 1525-5417$ & 1.0117 & $4.09 \times 10^{12}$ & $16.0 \pm 5.0$ & Wang, Manchester, \& Johnston 2007 \\
\hline 49 & B1524-39 & J1527-3931 & 2.4176 & $6.87 \times 10^{12}$ & $5.1 \pm 1.3$ & Basu, Mitra, \& Melikidze 2017 \\
\hline 50 & B $1530+27$ & $\mathrm{~J} 1532+2745$ & 1.1248 & $9.48 \times 10^{11}$ & $6.0 \pm 2.0$ & Weisberg et al. 1986 \\
\hline 51 & B1530-53 & J1534-5334 & 1.3689 & $1.41 \times 10^{12}$ & $\leq 0.25$ & Biggs 1992 \\
\hline 52 & B1540-06 & J1543-0620 & 0.7091 & $7.99 \times 10^{11}$ & $4.0 \pm 2.0$ & Naidu et al. 2017 \\
\hline \multirow[t]{2}{*}{53} & B1556-44 & $\mathrm{J} 1559-4438$ & 0.2571 & $5.18 \times 10^{11}$ & $\leq 0.01$ & Biggs 1992 \\
\hline & & & & & 0.24 & Basu, Mitra, \& Melikidze 2017 \\
\hline 54 & B1604-00 & J1607-0032 & 0.4218 & $3.64 \times 10^{11}$ & $\leq 0.1$ & Biggs 1992 \\
\hline 55 & B1612+07 & $\mathrm{J} 1614+0737$ & 1.2068 & $1.71 \times 10^{12}$ & $\leq 5.0$ & Weisberg et al. 1986 \\
\hline 56 & J1634-5107 & J1634-5107 & 0.5074 & $9.04 \times 10^{11}$ & $90.0 \pm 5.0$ & Young et al. 2015 \\
\hline 57 & J1639-4359 & J1639-4359 & 0.5876 & $9.50 \times 10^{10}$ & $\leq 0.1$ & Gajjar 2017 \\
\hline 58 & B1641-45 & J1644-4559 & 0.4551 & $3.06 \times 10^{12}$ & $\leq 0.4$ & Biggs 1992 \\
\hline 59 & B1642-03 & J1645-0317 & 0.3877 & $8.41 \times 10^{11}$ & $\leq 0.25$ & Ritchings 1976 \\
\hline 60 & $\mathrm{~J} 1648-4458$ & J1648-4458 & 0.6296 & $1.09 \times 10^{12}$ & 1.4 & Wang, Manchester, \& Johnston 2007 \\
\hline 61 & $\mathrm{~J} 1649+2533$ & $\mathrm{~J} 1649+2533$ & 1.0153 & $7.63 \times 10^{11}$ & $\leq 20.0$ & Redman \& Rankin 2009 \\
\hline \multirow[t]{2}{*}{62} & B1658-37 & $\mathrm{J} 1701-3726$ & 2.4546 & $5.29 \times 10^{12}$ & $14.0 \pm 2.0$ & Yang, Han, \& Wang 2014 \\
\hline & J1702-4428 & J1702-4428 & 2.1235 & $2.68 \times 10^{12}$ & $\begin{array}{l}19.0 \pm 6.0 \\
26.0 \pm 3.0\end{array}$ & \begin{tabular}{|l|} 
Gajjar 2017 \\
Wang, Manchester, \& Johnston 2007
\end{tabular} \\
\hline 64 & B1700-32 & J1703-3241 & 1.2118 & $9.05 \times 10^{11}$ & $1.6 \pm 0.4$ & Basu, Mitra, \& Melikidze 2017 \\
\hline \multirow[t]{2}{*}{65} & $\mathrm{~J} 1703-4851$ & $\mathrm{~J} 1703-4851$ & 1.3964 & $2.70 \times 10^{12}$ & 1.1 & Wang, Manchester, \& Johnston 2007 \\
\hline & & & & & 74.0 & Yang, Han, \& Wang 2014 \\
\hline 66 & B1706-16 & J1709-1640 & 0.6531 & $2.05 \times 10^{12}$ & $31.0 \pm 2.0,15.0$ & Naidu et al. 2018 \\
\hline 67 & J1715-4034 & $\mathrm{J} 1715-4034$ & 2.0722 & $2.53 \times 10^{12}$ & $\leq 6.0$ & Gajjar 2017 \\
\hline \multirow[t]{2}{*}{68} & B1713-40 & $\mathrm{J} 1717-4054$ & 0.8877 & $1.83 \times 10^{12}$ & $\overline{7} 7.0 \pm 5.0$ & Young et al. 2015 \\
\hline & & & & & $\geq 95.0$ & Wang, Manchester, \& Johnston 2007 \\
\hline 69 & B1718-32 & J1722-3207 & 0.4772 & $5.62 \times 10^{11}$ & $1.0 \pm 1.0$ & Naidu et al. 2017 \\
\hline 70 & J1725-4043 & $\mathrm{J} 1725-4043$ & 1.4651 & $2.05 \times 10^{12}$ & $\leq 70.0$ & Gajjar 2017 \\
\hline 71 & J1727-2739 & J1727-2739 & 1.2931 & $1.21 \times 10^{12}$ & $52.0 \pm 3.0$ & Wang, Manchester, \& Johnston 2007 \\
\hline 72 & B1727-47 & $\mathrm{J} 1731-4744$ & 0.8298 & $1.18 \times 10^{13}$ & $\leq 0.1$ & Biggs 1992 \\
\hline 73 & B1730-37 & J1733-3716 & 0.3376 & $2.28 \times 10^{12}$ & $52.4 \pm 3.5$ & Basu, Mitra, \& Melikidze 2017 \\
\hline 74 & $\mathrm{~J} 1738-2330$ & J1738-2330 & 1.9788 & $4.16 \times 10^{12}$ & $85.1 \pm 2.3$ & Gajjar 2017 \\
\hline 75 & B1737+13 & $\mathrm{J} 1740+1311$ & 0.8031 & $1.09 \times 10^{12}$ & $\leq 0.02$ & Weisberg et al. 1986 \\
\hline \multirow[t]{2}{*}{76} & B1738-08 & $\mathrm{J} 1741-0840$ & 2.0431 & $2.18 \times 10^{12}$ & $30.0 \pm 5.0$ & Gajjar et al. 2017 \\
\hline & & & & & $15.7 \pm 1.7,15.8 \pm 1.4$ & Basu, Mitra, \& Melikidze 2017 \\
\hline 77 & J1744-3922 & J1744-3922 & 0.1724 & $1.65 \times 10^{10}$ & $\leq 75.0$ & Faulkner et al. 2004 \\
\hline 78 & B1742-30 & $\mathrm{J} 1745-3040$ & 0.3674 & $1.99 \times 10^{12}$ & $\leq 17.5$ & Biggs 1992 \\
\hline 79 & B1747-46 & J1751-4657 & 0.7424 & $9.91 \times 10^{11}$ & $2.4 \pm 0.5$ & Basu, Mitra, \& Melikidze 2017 \\
\hline 80 & $\mathrm{~J} 1752+2359$ & $\mathrm{~J} 1752+2359$ & 0.4091 & $5.19 \times 10^{11}$ & $\leq 89.0$ & Gajjar 2017 \\
\hline 81 & B1749-28 & J1752-2806 & 0.5626 & $2.16 \times 10^{12}$ & $\leq 0.75$ & Ritchings 1976 \\
\hline 82 & $\mathrm{~J} 1752+2359$ & $\mathrm{~J} 1752+2359$ & 0.4091 & $5.19 \times 10^{11}$ & 81.0 & Yang, Han, \& Wang 2014 \\
\hline 83 & B1758-03 & $\mathrm{J} 1801-0357$ & 0.9215 & $1.77 \times 10^{12}$ & $27.7 \pm 1.3,26.1 \pm 2.6$ & Basu, Mitra, \& Melikidze 2017 \\
\hline 84 & $\mathrm{~J} 1808-0813$ & $\mathrm{~J} 1808-0813$ & 0.8760 & $1.05 \times 10^{12}$ & $1.28 \pm 1.3$ & Basu, Mitra, \& Melikidze 2017 \\
\hline 85 & B1809-173 & J1812-1718 & 1.2054 & $4.85 \times 10^{12}$ & $5.8 \pm 0.4$ & Wang, Manchester, \& Johnston 2007 \\
\hline
\end{tabular}


Table 4: Continuation of Table $2 \& 3$.

\begin{tabular}{|c|c|c|c|c|c|c|}
\hline & PSR Name & J-Name & $\begin{array}{l}\mathrm{P}_{\mathrm{s}} \\
(\mathrm{s})\end{array}$ & $\begin{array}{l}\mathrm{B}_{\mathrm{s}} \\
(\mathrm{G})\end{array}$ & $\begin{array}{l}\text { NF } \\
(\%)\end{array}$ & References \\
\hline 86 & B1813-36 & $\mathrm{J} 1817-3618$ & 0.3870 & $9.01 \times 10^{11}$ & $16.7 \pm 0.7$ & Basu, Mitra, \& Melikidze 2017 \\
\hline 87 & $\mathrm{~J} 1819+1305$ & $\mathrm{~J} 1819+1305$ & 1.0604 & $6.25 \times 10^{11}$ & $41.0 \pm 6.0$ & Yang, Han, \& Wang 2014 \\
\hline 88 & B1818-04 & $\mathrm{J} 1820-0427$ & 0.5981 & $1.97 \times 10^{12}$ & $\leq 0.25$ & Biggs 1992 \\
\hline 89 & $\mathrm{~J} 1820-0509$ & J1820-0509 & 0.3373 & $5.67 \times 10^{11}$ & $67.0 \pm 3.0$ & Wang, Manchester, \& Johnston 2007 \\
\hline 90 & B1819-22 & $\mathrm{J} 1822-2256$ & 1.8743 & $1.61 \times 10^{12}$ & $\begin{array}{l}10.0 \pm 2.0 \\
4.7 \pm 0.9\end{array}$ & $\begin{array}{l}\text { Naidu et al. 2017 } \\
\text { Basu, Mitra, \& Melikidze } 2017\end{array}$ \\
\hline 91 & B1821+05 & $\mathrm{J} 1823+0550$ & 0.7529 & $4.18 \times 10^{11}$ & $\begin{array}{l}5.5 \pm 0.7 \\
\leq 0.4\end{array}$ & $\begin{array}{l}\text {-do- } \\
\text { Weisberg et al. } 1986\end{array}$ \\
\hline 92 & $\mathrm{~J} 1831-1223$ & $\mathrm{~J} 1831-1223$ & 2.8580 & $3.99 \times 10^{12}$ & $4.0 \pm 1.0$ & Wang, Manchester, \& Johnston 2007 \\
\hline 93 & $\mathrm{~J} 1833-1055$ & J1833-1055 & 0.6336 & $5.85 \times 10^{11}$ & $7.0 \pm 2.0$ & Wang, Manchester, \& Johnston 2007 \\
\hline 94 & $\mathrm{~J} 1840-0840$ & $\mathrm{~J} 1840-0840$ & 5.3094 & $1.13 \times 10^{13}$ & $50.0 \pm 6.0$ & Gajjar et al. 2017 \\
\hline 95 & B $1839+09$ & $\mathrm{~J} 1841+0912$ & 0.3813 & $6.52 \times 10^{11}$ & $\leq 5.0$ & Weisberg et al. 1986 \\
\hline 96 & J1843-0211 & J1843-0211 & 2.0275 & $5.48 \times 10^{12}$ & $6.0 \pm 2.0$ & Wang, Manchester, \& Johnston 2007 \\
\hline 97 & B1842+14 & $\mathrm{J} 1844+1454$ & 0.3755 & $8.48 \times 10^{11}$ & $\leq 0.15$ & Weisberg et al. 1986 \\
\hline 98 & B1844-04 & $\mathrm{J} 1847-0402$ & 0.5978 & $5.63 \times 10^{12}$ & $3.0 \pm 1.0$ & Naidu et al. 2017 \\
\hline 99 & B1845-19 & $\mathrm{J} 1848-1952$ & 4.3082 & $1.01 \times 10^{13}$ & $27.0 \pm 6.0$ & Naidu et al. 2017 \\
\hline 100 & $\mathrm{~B} 1848+12$ & $\mathrm{~J} 1851+1259$ & 1.2053 & $3.77 \times 10^{12}$ & $\leq 54.0$ & Redman \& Rankin 2009 \\
\hline 101 & $\mathrm{~J} 1853+0505$ & $\mathrm{~J} 1853+0505$ & 0.9051 & $1.09 \times 10^{12}$ & $67.0 \pm 8.0$ & Young et al. 2015 \\
\hline 102 & B1857-26 & $\mathrm{J} 1900-2600$ & 0.6122 & $3.58 \times 10^{11}$ & $10.0 \pm 2.5$ & Ritchings 1976 \\
\hline 103 & $\mathrm{~J} 1901+0413$ & $\mathrm{~J} 1901+0413$ & 2.6631 & $1.89 \times 10^{13}$ & $\leq 6.0$ & Gajiar 2017 \\
\hline \multirow[t]{3}{*}{104} & J1901-0906 & J1901-0906 & 1.7819 & $1.73 \times 10^{12}$ & $29.0 \pm 4.0$ & Naidu et al. 2017 \\
\hline & & & & & 2.9 & Basu, Mitra, \& Melikidze 2017 \\
\hline & & & & & $5.6 \pm 0.7$ & - do- \\
\hline 105 & B $1907+03$ & $\mathrm{~J} 1910+0358$ & 2.3303 & $3.27 \times 10^{12}$ & $4.0 \pm 0.2$ & Weisberg et al. 1986 \\
\hline 106 & B1911-04 & J1913-0440 & 0.8259 & $1.85 \times 10^{12}$ & $\leq 0.5$ & Ritchings 1976 \\
\hline 107 & $\mathrm{~J} 1916+1023$ & $\mathrm{~J} 1916+1023$ & 1.6183 & $1.06 \times 10^{12}$ & $47.0 \pm 4.0$ & Wang, Manchester, \& Johnston 2007 \\
\hline 108 & $\mathrm{~B} 1917+00$ & $\mathrm{~J} 1919+0021$ & 1.2723 & $3.16 \times 10^{12}$ & $\leq 0.4$ & Rankin 1986 \\
\hline 109 & $\mathrm{~J} 1920+1040$ & $\mathrm{~J} 1920+1040$ & 2.2158 & $3.83 \times 10^{12}$ & $50.0 \pm 4.0$ & Wang, Manchester, \& Johnston 2007 \\
\hline 110 & B1918+19 & $\mathrm{J} 1921+1948$ & 0.8210 & $8.68 \times 10^{11}$ & $9.0,43.0$ & Rankin, Wright, \& Brown 2013 \\
\hline 111 & $\mathrm{~B} 1919+21$ & $\mathrm{~J} 1921+2153$ & 1.3373 & $1.36 \times 10^{12}$ & $\leq 0.25$ & Ritchings 1976 \\
\hline 112 & J1926-1314 & J1926-1314 & 4.8643 & $1.35 \times 10^{13}$ & $\sim 75.7 \pm 1.9$ & Rosen et al. 2013 \\
\hline 113 & B $1923+04$ & $\mathrm{~J} 1926+0431$ & 1.0741 & $1.64 \times 10^{12}$ & $\leq 5.0$ & Weisberg et al. 1986 \\
\hline 114 & B1929+10 & $\mathrm{J} 1932+1059$ & 0.2265 & $5.18 \times 10^{11}$ & $\leq 1.0$ & Ritchings 1976 \\
\hline 115 & $\mathrm{~B} 1933+16$ & $\mathrm{~J} 1935+1616$ & 0.3587 & $1.48 \times 10^{12}$ & $\leq 0.06$ & Biggs 1992 \\
\hline 116 & B $1942+17$ & $\mathrm{~J} 1944+1755$ & 1.9969 & $1.22 \times 10^{12}$ & $\leq 60.0$ & Lorimer, Camilo, \& Xilouris 2002 \\
\hline 117 & B1942-00 & J1945-0040 & 1.0456 & $7.57 \times 10^{11}$ & $21.0 \pm 1.0$ & Weisberg et al. 1986 \\
\hline 118 & B1944+17 & $\mathrm{J} 1946+1805$ & 0.4406 & $1.04 \times 10^{11}$ & $\begin{array}{l}55.0 \pm 5.0 \\
64.0 \pm 32.0\end{array}$ & \begin{tabular}{|l|} 
Yang, Han, \& Wang 2014 \\
Ritchings 1976
\end{tabular} \\
\hline 119 & B1946+35 & $\mathrm{J} 1948+3540$ & 0.7173 & $2.28 \times 10^{12}$ & $\leq 0.75$ & Ritchings 1976 \\
\hline 120 & B2003-08 & J2006-0807 & 0.5809 & $1.65 \times 10^{11}$ & $15.5 \pm 1.0$ & Basu, Mitra, \& Melikidze 2017 \\
\hline 121 & B2016+28 & $\mathrm{J} 2018+2839$ & 0.5580 & $2.91 \times 10^{11}$ & $1.0 \pm 3.0$ & Naidu et al. 2017 \\
\hline 122 & B2020+28 & $\mathrm{J} 2022+2854$ & 0.3434 & $8.16 \times 10^{11}$ & $0.2 \pm 1.6$ & Gajjar 2017 \\
\hline 123 & $\mathrm{~B} 2021+51$ & $\mathrm{~J} 2022+5154$ & 0.5292 & $1.29 \times 10^{12}$ & $1.4 \pm 0.7$ & Gajjar 2017 \\
\hline 124 & $\mathrm{~J} 2033+0042$ & $\mathrm{~J} 2033+0042$ & 5.0134 & $7.21 \times 10^{12}$ & $44-49,53-58$ & Lynch et al. 2013 \\
\hline \multirow[t]{2}{*}{125} & B2034+19 & $\mathrm{J} 2037+1942$ & 2.0744 & $2.08 \times 10^{12}$ & $44.0 \pm 4.0$ & Herfindal \& Rankin 2009 \\
\hline & & & & & $24.2 \pm 1.5$ & - do- \\
\hline
\end{tabular}


Table 5: Continuation of Table 2, $3 \& 4$.

\begin{tabular}{|c|c|c|c|c|c|c|}
\hline & PSR Name & J-Name & $\begin{array}{l}P_{s} \\
(s)\end{array}$ & $\begin{array}{l}B_{s} \\
(G)\end{array}$ & $\begin{array}{l}\mathrm{NF} \\
(\%)\end{array}$ & References \\
\hline 126 & B2044+15 & $\mathrm{J} 2046+1540$ & 1.1383 & $4.61 \times 10^{11}$ & $\leq 0.04$ & Weisberg et al. 1986 \\
\hline 127 & B2045-16 & $\mathrm{J} 2048-1616$ & 1.9616 & $4.69 \times 10^{12}$ & $\begin{array}{l}22.0 \pm 5.0 \\
5.5 \pm 0.2\end{array}$ & \begin{tabular}{|l|} 
Naidu et al. 2017 \\
Basu \& Mitra 2018
\end{tabular} \\
\hline 128 & $\mathrm{~B} 2053+36$ & $\mathrm{~J} 2055+3630$ & 0.2215 & $2.89 \times 10^{11}$ & $\leq 0.7$ & Weisberg et al. 1986 \\
\hline 129 & B2110+27 & $\mathrm{J} 2113+2754$ & 1.2028 & $1.78 \times 10^{12}$ & $\leq 30.0$ & Redman \& Rankin 2009 \\
\hline 130 & B2111+46 & $\mathrm{J} 2113+4644$ & 1.0147 & $8.62 \times 10^{11}$ & $21.0 \pm 4.0$ & Gajjar 2017 \\
\hline 131 & B2113+14 & $\mathrm{J} 2116+1414$ & 0.4402 & $3.61 \times 10^{11}$ & $\leq 1.0$ & Weisberg et al. 1986 \\
\hline 132 & $\mathrm{~B} 2122+13$ & $\mathrm{~J} 2124+1407$ & 0.6941 & $7.39 \times 10^{11}$ & $\leq 22.0$ & Redman \& Rankin 2009 \\
\hline 133 & B2154+40 & $\mathrm{J} 2157+4017$ & 1.5253 & $2.32 \times 10^{12}$ & $7.5 \pm 2.5$ & Ritchings 1976 \\
\hline 134 & $\mathrm{~J} 2208+5500$ & $\mathrm{~J} 2208+5500$ & 0.9332 & $2.58 \times 10^{12}$ & $\leq 7.5$ & Joshi et al. 2009 \\
\hline 135 & $\mathrm{~B} 2217+47$ & $\mathrm{~J} 2219+4754$ & 0.5385 & $1.23 \times 10^{12}$ & $\leq 2.0$ & Ritchings 1976 \\
\hline 136 & $\mathrm{~J} 2253+1516$ & $\mathrm{~J} 2253+1516$ & 0.7922 & $2.32 \times 10^{11}$ & $\leq 49.0$ & Redman \& Rankin 2009 \\
\hline 137 & $\mathrm{~B} 2303+30$ & $\mathrm{~J} 2305+3100$ & 1.5759 & $2.16 \times 10^{12}$ & 1.0 & Rankin 1986 \\
\hline 138 & B2310+42 & $\mathrm{J} 2313+4253$ & 0.3494 & $2.01 \times 10^{11}$ & $\leq 11.0$ & Redman \& Rankin 2009 \\
\hline 139 & $\mathrm{~B} 2315+21$ & $\mathrm{~J} 2317+2149$ & 1.4447 & $1.24 \times 10^{12}$ & $3.0 \pm 0.5$ & Weisberg et al. 1986 \\
\hline 140 & B2319+60 & $\mathrm{J} 2321+6024$ & 2.2565 & $4.03 \times 10^{12}$ & $29.0 \pm 1.0$ & Gajjar 2017 \\
\hline 141 & B2327-20 & $\mathrm{J} 2330-2005$ & 1.6436 & $2.79 \times 10^{12}$ & $12.0 \pm 1.0$ & Biggs 1992 \\
\hline 142 & J2346-0609 & J2346-0609 & 1.1815 & $1.28 \times 10^{12}$ & $\begin{array}{l}42.5 \pm 3.8 \\
28.7 \pm 1.8\end{array}$ & $\begin{array}{l}\text { Basu, Mitra, \& Melikidze } 2017 \\
\text { Basu, Mitra, \& Melikidze } 2017\end{array}$ \\
\hline
\end{tabular}


Table 6: $\mathrm{P}_{\mathrm{s}}$ and $\mathrm{B}_{\mathrm{s}}$ of known nulling pulsars for which no NF estimates are available.

\begin{tabular}{|c|c|c|c|c|c|}
\hline & PSR Name & J-Name & $\begin{array}{l}P_{s} \\
(s)\end{array}$ & $\begin{array}{l}\mathrm{B}_{\mathrm{s}} \\
(\mathrm{G})\end{array}$ & References \\
\hline 1 & $\mathrm{~J} 0229+20$ & $\mathrm{~J} 0229+20$ & 0.8069 & NA & Deneva et al. 2013 \\
\hline 2 & J0726-2612 & J0726-2612 & 3.4423 & $3.21 \times 10^{13}$ & Burke-Spolaor et al. 2012 \\
\hline 3 & B0853-33 & J0855-3331 & 1.2675 & $2.86 \times 10^{12}$ & Burke-Spolaor et al. 2012 \\
\hline 4 & J0941-39 & J0941-39 & 0.5868 & NA & Burke-Spolaor \& Bailes 2010 \\
\hline 5 & $\mathrm{~J} 0943+2253$ & $\mathrm{~J} 0943+2253$ & 0.5330 & $2.21 \times 10^{11}$ & Brinkman et al. 2018 \\
\hline 6 & $\mathrm{~J} 1012-5830$ & $\mathrm{~J} 1012-5830$ & 2.1336 & $9.07 \times 10^{12}$ & Burke-Spolaor et al. 2012 \\
\hline 7 & $\mathrm{~J} 1055-6905$ & $\mathrm{~J} 1055-6905$ & 2.9193 & $7.80 \times 10^{12}$ & Burke-Spolaor et al. 2012 \\
\hline 8 & B1056-57 & $\mathrm{J} 1059-5742$ & 1.1850 & $2.28 \times 10^{12}$ & Burke-Spolaor et al. 2012 \\
\hline 9 & $\mathrm{~J} 1129-53$ & $\mathrm{~J} 1129-53$ & 1.0629 & NA & Burke-Spolaor et al. 2012 \\
\hline 10 & B1131-62 & $\mathrm{J} 1133-6250$ & 1.0229 & $6.88 \times 10^{11}$ & Burke-Spolaor et al. 2012 \\
\hline 11 & B1154-62 & $\mathrm{J} 1157-6224$ & 0.4005 & $1.27 \times 10^{12}$ & Burke-Spolaor et al. 2012 \\
\hline 12 & $\mathrm{~J} 1225-6035$ & $\mathrm{~J} 1225-6035$ & 0.6263 & $4.30 \times 10^{11}$ & Burke-Spolaor et al. 2012 \\
\hline 13 & $\mathrm{~J} 1255-6131$ & $\mathrm{~J} 1255-6131$ & 0.6580 & $1.64 \times 10^{12}$ & Burke-Spolaor et al. 2012 \\
\hline 14 & $\mathrm{~J} 1307-6318$ & $\mathrm{~J} 1307-6318$ & 4.9624 & $1.04 \times 10^{13}$ & Burke-Spolaor et al. 2012 \\
\hline 15 & B1323-58 & $\mathrm{J} 1326-5859$ & 0.4780 & $1.26 \times 10^{12}$ & Burke-Spolaor et al. 2012 \\
\hline 16 & B1323-63 & J1326-6408 & 0.7927 & $1.59 \times 10^{12}$ & Burke-Spolaor et al. 2012 \\
\hline 17 & J1406-5806 & $\mathrm{J} 1406-5806$ & 0.2883 & $4.25 \times 10^{11}$ & Burke-Spolaor et al. 2012 \\
\hline 18 & $\mathrm{~J} 1423-6953$ & $\mathrm{~J} 1423-6953$ & 0.3334 & $7.04 \times 10^{11}$ & Burke-Spolaor et al. 2012 \\
\hline 19 & B1424-55 & $\mathrm{J} 1428-5530$ & 0.5703 & $1.10 \times 10^{12}$ & Burke-Spolaor et al. 2012 \\
\hline 20 & B1449-64 & $\mathrm{J} 1453-6413$ & 0.1795 & $7.10 \times 10^{11}$ & Burke-Spolaor et al. 2012 \\
\hline 21 & B1454-51 & $\mathrm{J} 1457-5122$ & 1.7483 & $3.08 \times 10^{12}$ & Burke-Spolaor et al. 2012 \\
\hline 22 & B1510-48 & $\mathrm{J} 1514-4834$ & 0.4548 & $6.56 \times 10^{11}$ & Burke-Spolaor et al. 2012 \\
\hline 23 & $\mathrm{~J} 1514-5925$ & $\mathrm{~J} 1514-5925$ & 0.1488 & $6.63 \times 10^{11}$ & Burke-Spolaor et al. 2012 \\
\hline 24 & B1555-55 & $\mathrm{J} 1559-5545$ & 0.9572 & $4.48 \times 10^{12}$ & Burke-Spolaor et al. 2012 \\
\hline 25 & $\mathrm{~J} 1624-4613$ & $\mathrm{~J} 1624-4613$ & 0.8712 & $2.33 \times 10^{11}$ & Burke-Spolaor et al. 2012 \\
\hline 26 & B1630-44 & $\mathrm{J} 1633-4453$ & 0.4365 & $1.67 \times 10^{12}$ & Burke-Spolaor et al. 2012 \\
\hline 27 & B1641-68 & $\mathrm{J} 1646-6831$ & 1.7856 & $1.76 \times 10^{12}$ & Burke-Spolaor et al. 2012 \\
\hline 28 & $\mathrm{~J} 1647-3607$ & $\mathrm{~J} 1647-3607$ & 0.2123 & $1.67 \times 10^{11}$ & Burke-Spolaor et al. 2012 \\
\hline 29 & $\mathrm{~J} 1649-4349$ & $\mathrm{~J} 1649-4349$ & 0.8707 & $1.98 \times 10^{11}$ & Burke-Spolaor et al. 2012 \\
\hline 30 & B1650-38 & $\mathrm{J} 1653-3838$ & 0.3050 & $9.33 \times 10^{11}$ & Burke-Spolaor et al. 2012 \\
\hline 31 & $\mathrm{~J} 1707-4729$ & J1707-4729 & 0.2665 & $6.53 \times 10^{11}$ & Burke-Spolaor et al. 2012 \\
\hline 32 & $\mathrm{~J} 1736-2457$ & $\mathrm{~J} 1736-2457$ & 2.6422 & $3.05 \times 10^{12}$ & Burke-Spolaor et al. 2012 \\
\hline 33 & $\mathrm{~J} 1741-3016$ & $\mathrm{~J} 1741-3016$ & 1.8938 & $4.18 \times 10^{12}$ & Burke-Spolaor et al. 2012 \\
\hline 34 & $\mathrm{~J} 1742-4616$ & $\mathrm{~J} 1742-4616$ & 0.4124 & $1.19 \times 10^{11}$ & Burke-Spolaor et al. 2012 \\
\hline 35 & $\mathrm{~J} 1749+16$ & $\mathrm{~J} 1749+16$ & 2.3117 & NA & Deneva et al. 2016 \\
\hline 36 & $\mathrm{~J} 1750+07$ & $\mathrm{~J} 1750+07$ & 1.9088 & NA & Deneva et al. 2016 \\
\hline 37 & B1747-31 & $\mathrm{J} 1750-3157$ & 0.9104 & $4.28 \times 10^{11}$ & Burke-Spolaor et al. 2012 \\
\hline 38 & $\mathrm{~J} 1757-2223$ & $\mathrm{~J} 1757-2223$ & 0.1853 & $3.85 \times 10^{11}$ & Burke-Spolaor et al. 2012 \\
\hline 39 & $\mathrm{~J} 1758-2540$ & $\mathrm{~J} 1758-2540$ & 2.1073 & $1.83 \times 10^{12}$ & Burke-Spolaor et al. 2012 \\
\hline 40 & B1806-21 & $\mathrm{J} 1809-2109$ & 0.7024 & $1.66 \times 10^{12}$ & Burke-Spolaor et al. 2012 \\
\hline 41 & $\mathrm{~J} 1819-1458$ & $\mathrm{~J} 1819-1458$ & 4.2632 & $5.01 \times 10^{13}$ & Burke-Spolaor et al. 2012 \\
\hline 42 & J1823-1126 & J1823-1126 & 1.8465 & $8.31 \times 10^{12}$ & Burke-Spolaor et al. 2012 \\
\hline 43 & B1822-14 & $\mathrm{J} 1825-1446$ & 0.2792 & $2.55 \times 10^{12}$ & Burke-Spolaor et al. 2012 \\
\hline 44 & $\mathrm{~J} 1827-0750$ & $\mathrm{~J} 1827-0750$ & 0.2705 & $6.54 \times 10^{11}$ & Burke-Spolaor et al. 2012 \\
\hline 45 & $\mathrm{~J} 1830-1135$ & $\mathrm{~J} 1830-1135$ & 6.2216 & $1.74 \times 10^{13}$ & Burke-Spolaor et al. 2012 \\
\hline
\end{tabular}


Table 7: Continuation of Table 6

\begin{tabular}{|c|c|c|c|c|c|}
\hline & PSR Name & J-Name & $\begin{array}{l}P_{s} \\
(s)\end{array}$ & $\begin{array}{l}\mathrm{B}_{\mathrm{s}} \\
(\mathrm{G})\end{array}$ & References \\
\hline 46 & B1834-06 & $\mathrm{J} 1837-0653$ & 1.9058 & $1.23 \times 10^{12}$ & Burke-Spolaor et al. 2012 \\
\hline 47 & $\mathrm{~J} 1837-1243$ & $\mathrm{~J} 1837-1243$ & 1.8760 & $8.38 \times 10^{12}$ & Burke-Spolaor et al. 2012 \\
\hline 48 & $\mathrm{~J} 1840-1419$ & J1840-1419 & 6.5976 & $6.54 \times 10^{12}$ & Burke-Spolaor et al. 2012 \\
\hline 49 & $\mathrm{~J} 1841-0310$ & $\mathrm{~J} 1841-0310$ & 1.6577 & $7.54 \times 10^{11}$ & Burke-Spolaor et al. 2012 \\
\hline 50 & $\mathrm{~J} 1852-0635$ & $\mathrm{~J} 1852-0635$ & 0.5242 & $2.78 \times 10^{12}$ & Burke-Spolaor et al. 2012 \\
\hline 51 & J1854-1557 & J1854-1557 & 3.4532 & $3.99 \times 10^{12}$ & Burke-Spolaor et al. 2011 \\
\hline 52 & J1857-1027 & $\mathrm{J} 1857-1027$ & 3.6872 & $6.31 \times 10^{12}$ & Burke-Spolaor et al. 2012 \\
\hline 53 & $\mathrm{~J} 1935+1159$ & $\mathrm{~J} 1935+1159$ & 1.9398 & $1.37 \times 10^{12}$ & Brinkman et al. 2018 \\
\hline 54 & B2043-04 & $\mathrm{J} 2046-0421$ & 1.5469 & $1.53 \times 10^{12}$ & Naidu et al. 2017 \\
\hline 55 & $\mathrm{~J} 2050+1259$ & $\mathrm{~J} 2050+1259$ & 1.2210 & $7.94 \times 10^{11}$ & Brinkman et al. 2018 \\
\hline
\end{tabular}

Table 8: $P_{s}$ and $B_{s}$ of known Intermittent Pulsars.

\begin{tabular}{|c|c|c|c|c|c|}
\hline & PSR Name & J-Name & $\begin{array}{l}P_{s} \\
(s)\end{array}$ & $\begin{array}{l}\mathrm{B}_{\mathrm{s}} \\
(\mathrm{G})\end{array}$ & References \\
\hline 1 & J1107-5907 & J1107-5907 & 0.2528 & $4.83 \times 10^{10}$ & Meyers et al. 2018 \\
\hline 2 & $\mathrm{~J} 1832+0029$ & $\mathrm{~J} 1832+0029$ & 0.5339 & $9.09 \times 10^{11}$ & Lorimer et al. 2012 \\
\hline 3 & $\mathrm{~J} 1839+15$ & $\mathrm{~J} 1839+15$ & 0.5492 & $3.83 \times 10^{12}$ & Surnis et al. 2013 \\
\hline 4 & $\mathrm{~J} 1841-0500$ & $\mathrm{~J} 1841-0500$ & 0.9129 & $5.80 \times 10^{12}$ & Camilo et al. 2012 \\
\hline 5 & $\mathrm{~J} 1910+0517$ & $\mathrm{~J} 1910+0517$ & 0.3080 & $4.80 \times 10^{11}$ & Lyne et al. 2017 \\
\hline 6 & $\mathrm{~J} 1929+1357$ & J1929+1357 & 0.8669 & $1.80 \times 10^{12}$ & Lyne et al. 2017 \\
\hline 7 & B $1931+24$ & $\mathrm{~J} 1933+2421$ & 0.8137 & $2.60 \times 10^{12}$ & Kramer et al. 2006a \\
\hline
\end{tabular}

